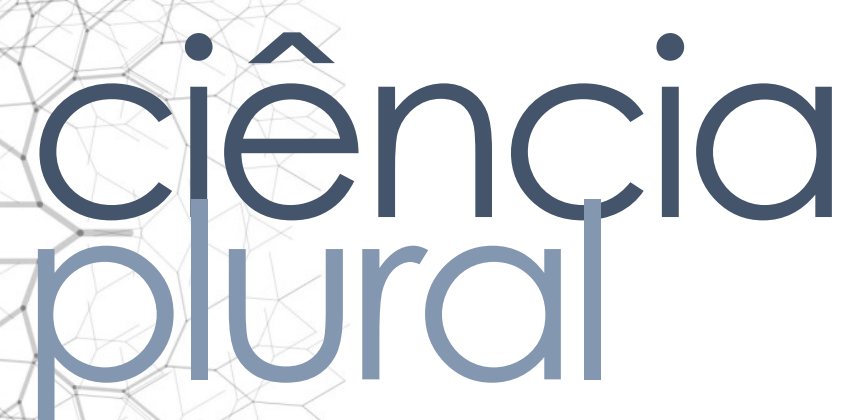

\title{
NECROSE MANDIBULAR ASSOCIADA AO USO DE BIFOSFONATO: RELATO DE CASO
}

Mandibular necrosis associated with biphosphonate use: case report

Necrosis mandibular asociada al uso de bifosfonato: relato de caso

Erasmo Freitas de Souza Junior • Universidade do Estado do Rio Grande do NorteUERN • Mestre pelo Programa de Pós-Graduação em Saúde e Sociedade • E-mail: erasmo_jn@hotmail.com

Janielma Azevedo Silva • UERN• Mestranda do Programa de Pós-Graduação em Saúde e Sociedade • E-mail: janielma.azevedo@hotmail.com

Maria do Socorro Soares $\bullet$ UERN • Graduanda do Curso de Odontologia • E-mail: airamsoares76@gmail.com

Hécio Henrique Araújo de Morais • UERN• Professor do Programa de PósGraduação em Saúde e Sociedade •E-mail: heciomorais@hotmail.com

Autor responsável pela correspondência:

Erasmo Freitas de Souza Junior • E-mail: erasmo_jn@hotmail.com 


\section{RESUMO}

Introdução: Os bifosfonatos são medicamentos que objetivam reduzir a reabsorção óssea por provocarem a apoptose de osteoclastos. Por essa razão, são frequentemente empregados em pacientes com osteoporose e no tratamento do câncer. Contudo, apesar dos benefícios associados ao tratamento com bifosfonatos, esses medicamentos vêm sendo relacionados a osteonecrose maxilo-mandibular, materializando-se como um preocupante quadro de interesse à saúde pública. Objetivo: Apresentar um caso clínico de osteonecrose mandibular associada ao uso de bifosfanato, após extração de um elemento dentário, bem como contribuir com as discussões sobre as possibilidades terapêuticas desta enfermidade. Descrição do caso: Paciente do sexo masculino, com 59 anos idade, encaminhado ao serviço de Cirurgia e Traumatologia Bucomaxilofacial, dois meses após a extração do elemento dentário 45. O diagnóstico foi de Osteonecrose Maxilomandibular Relacionada a Bifosfonatos, sendo realizado, inicialmente, a remoção dos traumas existentes, como também o cuidado com a área exposta. Posteriormente, optou-se pela remoção do segmento ósseo necrótico. Conclusões: A complexa fisiopatologia do tipo de osteonecrose estudada exige dos Cirurgiões Dentistas a tomada de medidas em tempo oportuno e que cause o mínimo transtorno à vida dos pacientes. Assim, deve-se avaliar o estado da lesão, havendo indicação de terapia conservadora para os achados precoces. O desbridamento cirúrgico é aconselhado para o tratamento da doença avançada e não responsiva.

Palavras-Chave: Bifosfonatos; Osteonecrose; Mandíbula.

\section{ABSTRACT}

Introduction: Bisphosphonates are medications that aim to reduce bone resorption by causing osteoclast apoptosis. For this reason, they are often used in patients with osteoporosis and in the treatment of cancer. However, despite the benefits associated with treatment with bisphosphonates, these drugs have been linked to maxillomandibular osteonecrosis, materializing as a worrying picture of public health concern. Objective: To present a clinical case of mandibular osteonecrosis associated with the use of bisphosphate after extraction of a dental element, as well as to contribute to the discussions about the therapeutic possibilities of this disease. Case description: Male patient, 59 years old, referred to the Buccomaxillofacial Surgery and Traumatology service, two months after extraction of the dental element 45 . The diagnosis was Bisphosphonate-Related Maxillomandibular Osteonecrosis, initially performed, the removal of existing traumas, as well as care for the exposed area. Subsequently, it was decided to remove the necrotic bone segment. Conclusions: The complex pathophysiology of the type of osteonecrosis studied requires that dental urgeons take measures in a timely manner and cause the least disturbance to patients' ives. Thus, the condition of the lesion should be assessed, with an indication for nservative therapy for early findings. Surgical debridement is recommended for the atment of advanced and unresponsive diseases.

words: Bisphosphonates; Osteonecrosis; Jaw. 


\section{RESUMEN}

Introducción: Los bisfosfonatos son medicamentos que tienen como objetivo reducir la resorción ósea al causar apoptosis de osteoclastos. Por esta razón, a menudo se usan en pacientes con osteoporosis y en el tratamiento del cáncer. Sin embargo, a pesar de los beneficios asociados con el tratamiento con bisfosfonatos, estos medicamentos se han relacionado con la osteonecrosis maxilomandibular, materializándose como un escenario preocupante de interés para la salud pública. Objetivo: Presentar un caso clínico de osteonecrosis mandibular asociada con el uso de bisfosfanato después de la extracción de un elemento dental, así como contribuir a las discusiones sobre las posibilidades terapéuticas de esta enfermedad. Descripción del caso: un paciente masculino de 59 años fue derivado del Departamento de Traumatología y Cirugía Buccomaxilofacial después de meses de extracción dental 45. El diagnóstico era de Osteonecrosis Maxilomandibular Relacionada con Bifosfonatos, inicialmente eligiendo eliminar los traumas existente, bien como del cuidado con el área expuesta. Posteriormente, elegimos eliminar el segmento óseo necrótico. Conclusiones: La compleja fisiopatología del tipo de osteonecrosis estudiada requiere que los cirujanos dentales tomen las medidas adecuadas y causen el menor inconveniente a la vida de los pacientes. Por lo tanto, se debe evaluar el estado de la lesión, con terapia conservadora indicada para hallazgos tempranos. El desbridamiento quirúrgico se recomienda para el tratamiento de enfermedades avanzadas y que no responden.

Palabras clave: Bisfosfonatos; Osteonecrosis; Mandíbula. 


\section{Introdução}

Os Bifosfonatos (BF) (incluindo o etidronato, tiludronato, clodronato, pamidronato, alendronato, ibandronato, risedronato e zoledronato) são drogas que se ligam a cristais de hidroxiapatita e são preferencialmente absorvidos pelos osteoclastos, provocando sua lise e inibindo assim a reabsorção óssea 1;2;3;4;5;6.

Pela sua característica de aumentar a densidade óssea, mediante alteração do equilíbrio entre a reabsorção e a deposição óssea, em favor da deposição, os Bifosfonatos são amplamente utilizados para o manejo de doenças, como a osteoporose ou neoplasias ósseas malignas, objetivando evitar fraturas patológicas e reduzir a dor $1 ; 7 ; 3 ; 8 ; 2 ; 4 ; 9 ; 6 ; 10$.

Podendo ser nitrogenados ou não, os que contem nitrogênio (incluindo o pamidronato, alendronato, ibandronato, risedronato e zoledronato) são mais potentes, afetando a via do mevalonato pela inibição da farnesil difosfato sintase, uma enzima importante na síntese do farnesil pirofosfato e para a biossíntese do colesterol, que acarreta o bloqueio da síntese de proteínas essenciais para o tráfego de vesículas e manutenção do citoesqueleto dos osteoclastos, inibindo fortemente a reabsorção óssea osteoclástica. Os que não contêm nitrogênio, como clodronato, etidronato e tiludronato, exercem seus efeitos nos osteoclastos competindo com o adenosina trifosfato como substrato, impactando significativamente sua função de reabsorção óssea ${ }^{1 ; 7 ; 3 ; 11 .}$

Embora o uso de Bifosfonatos seja benéfico para o tratamento de câncer e doenças esqueléticas, desde 2003, após a publicação de uma série de casos com 36 lesões ósseas maxilomandibulares em pacientes submetidos a tratamento com pamidronato ou zolendronato, a Osteonecrose Maxilomandibular Relacionada a Bifosfonatos (OMRB) tem sido relatada como um efeito adverso grave dessas drogas, especialmente se utilizadas em altas doses e por tempo prolongado, caracterizando-se pela morte e destruição progressiva dos tecidos ósseos gnáticos 3;12;2;4;13;14;15;16;1;9;5;6;10.

Em 2007 a Associação Americana de Cirurgiões Orais e Maxilofaciais (COM), definiu a OMRB como uma área destrutiva de osso exposto, podendo ser dada através de uma fístula intra ou extraoral na região maxilofacial, com evolução 8 semanas sem cicatrização, em pacientes expostos a medicamentos 
antirreabsortivos e sem histórico de radioterapia nos maxilares. Uma vez estabelecida a lesão, o seu curso é de difícil previsibilidade e o tratamento é complexo, principalmente nos casos mais avançados, caracterizando-se como uma grave condição de saúde pública. Tal definição foi reforçada por diversas outras publicações ${ }^{17 ; 2 ; 8 ; 1 ; 9 ; 18 ; 19 ; 6 ; 10 ; 16 ; 20 . ~}$

Intervenções cirúrgicas, como a extração dentária, foram relatadas como evento desencadeador de maior relevância para OMRB. Contudo, dados recentes sugerem que, doenças infecciosas odontogênicas, como cárie com comprometimento do periápice, doenças periodontais e instalação de implante dentário também podem ser responsáveis pelo surgimento dessa enfermidade $12 ; 7 ; 4 ; 13 ; 14 ; 8 ; 3 ; 18 ; 5 ; 10 ; 11 ; 16$.

Dentre outros fatores de risco relacionados estão os processos malignos, que recebem tratamento com corticosteroides, imunossupressores e antiangiogênicos, idade elevada, diabetes, osteoporose, má higiene bucal, uso de próteses mal adaptadas, tabagismo e quimioterapia 7;14;8;1;9;3;18;10;11;16.

Com o intuito de direcionar a abordagem dada aos indivíduos com OMRB, a AACOM propôs uma classificação, considerando estágios da doença e condutas a serem tomadas, trazendo os seguintes aspectos ${ }^{10}$ :

Estágio 0 - Ausência de exposição de osso necrótico, mas presença de alterações radiográficas, podendo apresentar mobilidade dentária, odontalgia sem causa aparente e fístula que não é associada com necrose pulpar ou conexa com doença periodontal crônica. A terapêutica consiste em manejo sistêmico, incluindo o uso de medicação para dor e antibióticos ${ }^{10}$;

Estágio 1 - Exposição de osso necrótico, assintomático e sem sinais de infecção. Preconiza-se enxaguatório bucal antibacteriano, acompanhamento clínico e revisão das indicações para continuação da terapia com bifosfonatos ${ }^{10}$;

Estágio 2 - Osteonecrose com exposição óssea e sinais clínicos de infecção. O tratamento deve ser sintomático, com controle da dor, desbridamento, antibioticoterapia local e sistêmica ${ }^{10}$;

stágio 3 - Osso necrótico exposto estendendo-se além do osso alveolar (borda inferior ramo na mandíbula, seio maxilar e processo zigomático da maxila) resultando em atura patológica, fístula extra-oral, comunicação oro-antral, ou osteólise, 
estendendo-se para borda inferior da mandíbula ou assoalho do seio. Emprega-se enxaguatório bucal antibacteriano, antibioticoterapia sistêmica, controle da dor e desbridamento cirúrgico ${ }^{10}$.

Mediante justificáveis questões éticas, há carência de estudos e ensaios clínicos em humanos sobre a temática, o que eleva o valor da documentação de casos clínicos na construção do conhecimento sobre a OMRB. Desse modo, este trabalho objetiva relatar um caso de OMRB após extração dentária, assim como a conduta terapêutica tomada, descrevendo e discutindo os aspectos táticos, teóricos e técnica envolvida.

\section{Relato de Caso}

Paciente do sexo masculino, com 59 anos de idade, foi encaminhado ao serviço de Cirurgia e Traumatologia Bucomaxilofacial do Curso de Odontologia da Universidade do Estado do Rio Grande do Norte (UERN), dois meses após a extração do elemento dentário 45 .

Ao exame intra-oral notou-se exposição óssea na região de rebordo alveolar mandibular direito com discreta presença de supuração (Figura 1B). A radiografia panorâmica mostrou imagem compatível com retardo do processo de reparo alveolar (Figura 1A). Durante a anamnese o paciente referiu estar sob tratamento médico de um câncer pulmonar, fazendo uso de BF (pamidronato) por dois anos, além de outros quimioterápicos. A cintilografia também demonstrou área de hipercaptação em fêmur esquerdo (Figura 1C), o que mais tarde revelou ser uma metástase secundária do câncer de pulmão, confirmado por meio de imunohistoquímica. O paciente não relatou outras patologias de base pré-existentes, como doenças cardiovasculares e diabetes mellitus, ou uso de esteroide e outros medicamentos conhecidos por afetar o metabolismo ósseo, contudo, afirmou ser fumante.

Foi proposto o diagnóstico de OMRB, sendo realizado, a priori, a remoção dos traumas existentes, desaconselhando-se o uso da prótese e o hábito de fumar, bem omo o cuidado com a área exposta, com reforço na higienização (escovação e uso de orexidina $0,12 \%$ ). Nos períodos em que houve exacerbação da infecção o paciente foi entado a utilizar amoxicilina 500mg com ácido clavulânico $125 \mathrm{mg}$, a cada 8 horas. o avanço da necrose optou-se pela remoção do segmento ósseo necrótico, sob 


\section{ciência
purala}

anestesia local, tomando-se o cuidado de remover todo o fragmento ósseo necrosado (Figura 2A), bem como permitir a sutura do retalho sem pontos de tensão. $O$ paciente permaneceu sob acompanhamento por 1 ano, sem maiores complicações (Figura 2B).

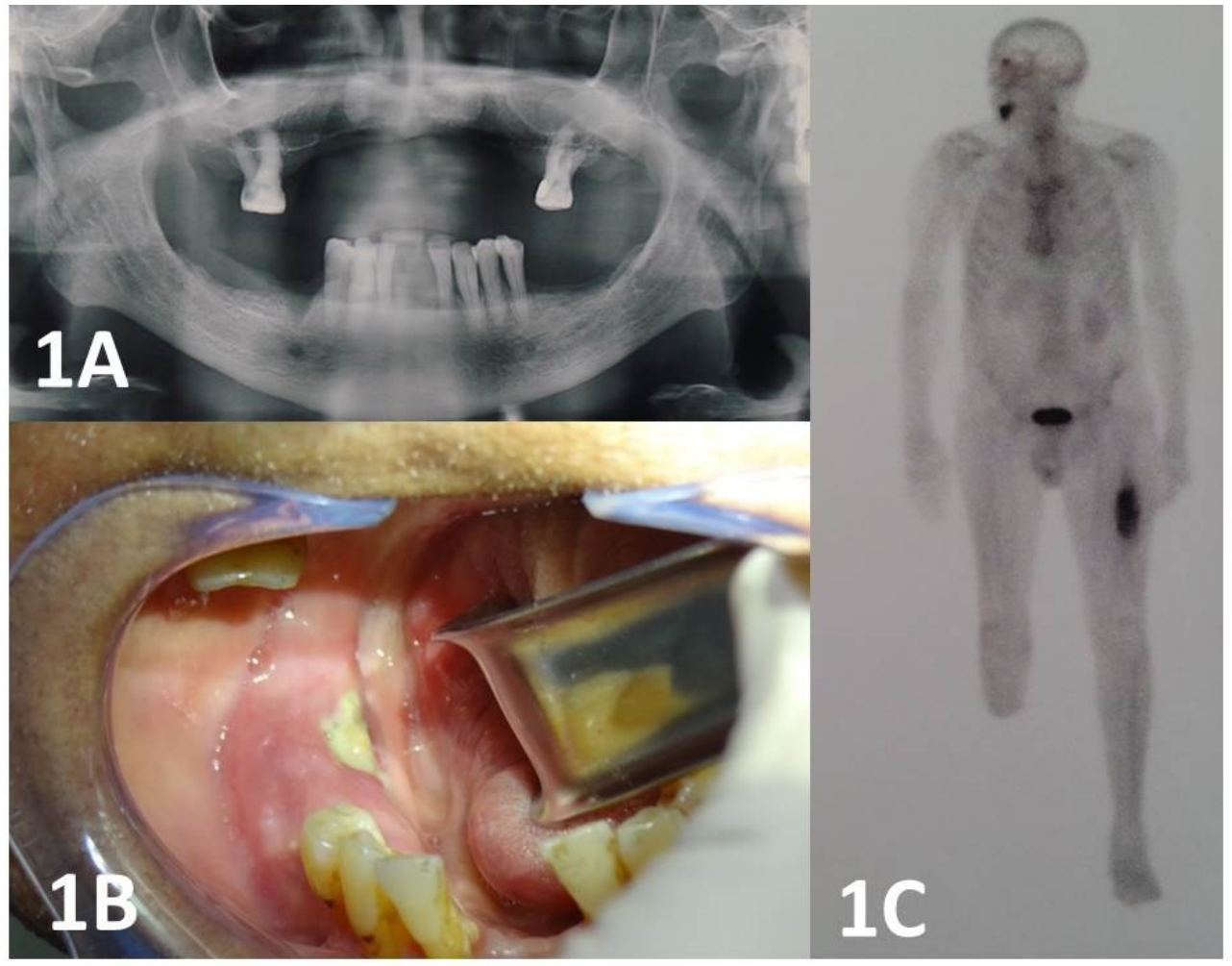

Figura 1: 1A, Aspecto radiográfico após exodontia do elemento 45. 1B, Aspecto do osso necrótico exposto (lado direito da mandíbula). 1C, Cintilografia mostrando área de hipercaptação no fêmur esquerdo. 


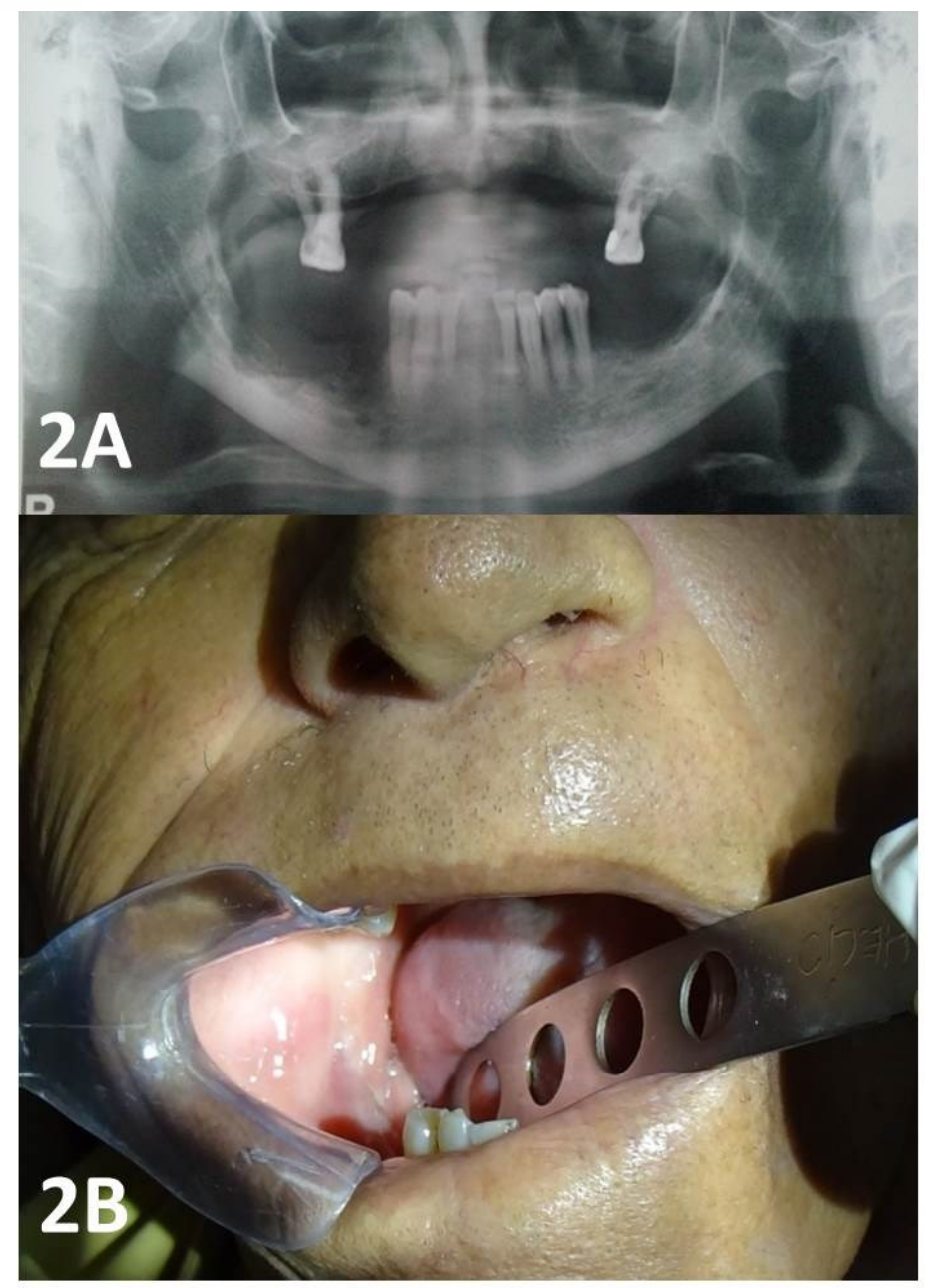

Figura 2: 2A, Aspecto radiográfico após remoção do osso necrótico. 2B, Aspecto clínico pósoperatório de 1 ano, observa-se o fechamento da ferida.

\section{Discussão}

Mediante a recente e crescente preocupação com a OMRB, séries e relatos de casos, bem como estudos envolvendo animais, vêm sendo realizados a fim de se obter uma melhor compreensão da patogênese, assim como o tratamento mais eficiente.

Como primeiro relato de OMRB, uma série de 36 casos de exposição óssea maxilomandibular, que não respondiam à cirurgia ou tratamentos medicamentosos, tiveram como ponto em comum a utilização de Bifosfonatos. Vinte e quatro receberam pamidronato de $90 \mathrm{mg}$ IV mensalmente, seis receberam zoledronato $4 \mathrm{mg}$ IV mensalmente no momento da apresentação, contudo, já haviam recebido terapia prévia com pamidronato e 6 receberam apenas zoledronato $4 \mathrm{mg}$ IV mensalmente. 
Dezoito pacientes receberam esses medicamentos para tratamento relacionado ao mieloma múltiplo, dezessete estavam relacionados ao carcinoma de mama metastático e um para osteoporose. A maioria dos pacientes apresentava necrose óssea na mandíbula $29(80,5 \%)$, na maxila foram 5 casos $(14 \%)$ e em ambos os ossos 2 casos $(5,5 \%)$. A exodontia foi a causa relacionada em $28(77,7 \%)$ pacientes, os 8 pacientes restantes $(22,3 \%)$ desenvolveram OMRB espontaneamente. Os autores justificaram as exposições espontâneas sugerindo o comprometimento periodontal como fator associado. Quanto ao tratamento, foi recomendado controle da progressão com antibióticos do tipo penicilina (eritromicinas ou tetraciclinas se alérgicos à penicilina), uso de clorexidina $0,12 \%$ e desbridamento secundário ${ }^{15}$.

Esta série de casos teve como pontos em comum com o aqui exposto as características clínicas, o uso de BF nitrogenado para tratamento de câncer, a predileção pela mandíbula como área afetada, a exodontia como principal causa relacionada e a conduta terapêutica para controlar a situação.

Apesar de que até o momento a compreensão do mecanismo e da patogênese ainda é insuficiente para definir o tratamento mais eficaz da OMRB, a conduta de eleição tem consistido em terapia conservadora, incluindo alívio da dor, o uso de antibióticos tópicos e sistêmicos para limitar a infecção secundária e prevenir a extensão do osso exposto, com sequestrectomia localizada conservadora e desbridamento cirúrgico para o tratamento de doença avançada e não responsiva, sendo necessárias imagens pré-operatórias precisas para definir áreas de necrose e delinear as margens de ressecção. O presente caso caracteriza um estágio 2 na classificação da Associação Americana de Cirurgiões Orais e Maxilofaciais e corrobora com os achados na literatura ao grupo de risco, fator desencadeante, tempo decorrido para o surgimento da lesão e sequência de tratamento instituída, partindo de uma terapêutica conservadora para uma abordagem mais invasiva a medida que a conduta inicial não fornece resultados satisfatórios ${ }^{12 ; 2 ; 4 ; 18 ; 10 ; 11 ; 16 ; 20 . ~}$

Em um estudo retrospectivo, um total de 194 pacientes tratados com Bifosfonatos intravenosos durante um período de 7 anos foram analisados. Desses 25 pacientes $(12,9 \%)$ sofreram OMRB, sendo a dor a complicação mais notável, relatada por $80 \%$ dos pacientes, seguida por espículas ósseas $(24 \%)$, abscessos $(24 \%)$ e, em 
menor grau, comunicação oroantral (4\%) e fístula extraoral (4\%). A idade média dos pacientes foi de 68,9 anos. Não houve diferenças estatisticamente significativas quanto ao sexo. O motivo da terapia com Bifosfonatos foi câncer. Os pacientes que foram submetidos a extrações durante o tratamento, com exceção de um, desenvolveram OMRB. Quase 50\% das necroses estavam localizados na mandíbula. Quanto aos fatores tóxicos relacionados, dos pacientes com OMRB, um total de 76\% não eram fumantes e não consumiam bebidas alcoólicas ${ }^{8}$.

Neste estudo foi possível notar as similaridades com o caso aqui exposto no tocante ao tratamento a longo prazo (o BF tem uma meia-vida esquelética de 8 a 15 anos $^{2 ; 11 ; 16 ; 20}$ ), sinais e sintomas da lesão, idade avançada dos pacientes, a casuística para o tratamento com Bifosfonatos, a predileção pela mandíbula e o hábito de fumar, em alguns casos.

Uma revisão sistemática referente à OMRB apontou que a incidência dessa lesão é maior em pacientes oncológicos, mediante as altas doses usadas em intervalos frequentes, com a maioria das séries de casos descrevendo a ocorrência em locais de cirurgia oral prévia, com fístulas intra e extraorais podendo se desenvolver quando o osso se infecta secundariamente. O uso de glicocorticóides, higiene bucal deficiente, inflamação crônica, diabetes mellitus, próteses mal ajustadas, bem como outras drogas, incluindo agentes antiangiogênicos, se apresentam como fator de risco, sendo estratégias de prevenção a eliminação ou estabilização de doenças orais antes do início de agentes antirreabsortivos, bem como a manutenção de uma adequada higiene bucal. O uso de tomografia computadorizada de feixe cônico, ressonância magnética, tomografia multi slice e tomografia por emissão de pósitrons são artifícios que podem ser utilizados, embora radiografias convencionais possam ser suficientes em alguns casos. A terapia conservadora inclui antibióticos tópicos e sistêmicos, sendo bemsucedida a indicação do desbridamento cirúrgico na doença avançada não responsiva, recomendando-se a cobertura total da loja cirúrgica por meio de retalho sem tensão. Para terapias experimentais incluem-se transplante intralesional de célulastronco de medula óssea e o uso de laser de baixa potência ${ }^{18}$. 
Neste último trabalho foi possível encontrar concordância com o relato de caso em questão, aqui exposto, no que diz respeito aos fatores associados. Ser paciente oncológico, ter sofrido cirurgia oral prévia, utilizar prótese mal adaptada, ser fumante, quanto à terapêutica abordada com o desaconselhamento do uso da prótese e o hábito de fumar, bem como o cuidado com a higienização, antibioticoterapia local e sistêmica, e remoção do segmento ósseo necrótico com a sutura do retalho sem pontos de tensão.

Outro ponto de destaque é que o aparecimento da OMRB, no presente caso, se deu após a extração dentária, contudo a maioria das extrações dentárias são realizadas como resultado de doenças infecciosas odontogênicas graves. Sendo as relações causais entre infecção, extração dentária e a OMRB ainda não amplamente conhecidas $^{12}$.

Objetivando desenvolver um modelo reprodutível para OMRB, foi realizado um estudo com oitenta ratos divididos em dois grupos. Um recebendo o tratamento com zoledronato IV, $80 \mu \mathrm{g} / \mathrm{kg} /$ semana durante 13 semanas e outro sendo grupo controle. Na décima terceira semana, o primeiro molar inferior esquerdo foi extraído, seguido pelo segundo molar uma semana depois, todos os animais tratados com zoledronato desenvolveram grave OMRB. Segundo os autores, a extração cirúrgica por si só precipitou a necrose óssea em animais tratados com zoledronato, eliminando a toxicidade de órgãos relacionados ao medicamento e a necessidade de induzir infecção pré-existente ou comorbidade como fator necessário para o surgimento da OMRB, sugerindo assim que a necrose óssea é provavelmente devida a um efeito direto da droga no osso ${ }^{13}$.

Em outro estudo com ratos, foi injetado altas doses de ácido zoledrônico, assim como realizada a exposição pulpar (EP) dos molares inferiores para induzir a doença periapical. Análises radiográficas e histológicas foram realizadas após 8 semanas. As lesões periapicais foram maiores nos camundongos não tratados com Bifosfonatos. Contudo, espessamento da lâmina dura, depósito ósseo periosteal e aumento da densidade trabecular foram observadas nos animais tratados com BF. Histologicamente, osteonecrose, espessamento periosteal, aposição óssea periosteal, migração epitelial e exposição óssea estavam presentes nos animais 
tratados com BF na presença de doença periapical. Embora 88\% dos animais tratados com BF apresentassem áreas de osteonecrose no local da doença dentária, apenas 33\% desenvolveram exposição óssea. Os autores sugeriram que a exposição óssea não é um pré-requisito para a necrose óssea ${ }^{3}$.

Ainda na investigação do papel da doença periapical na indução da OMRB, uma pesquisa usou camundongos ovariectomizados, em que oito semanas após o início da administração de ácido zoledrônico, uma operação de EP foi realizada no primeiro molar inferior direito para induzir a periodontite periapical, ficando o dente sem EP contralateral como controle. Clinicamente nenhum animal exibiu sinais clínicos de infecção, lesão da mucosa oral ou exposição óssea. Contudo, em análise de micro tomografia computadorizada, observou-se que o tratamento com ácido zoledrônico reduziu a absorção óssea resultante da doença periapical, mas aumentou o risco de desenvolver OMRB ${ }^{12}$.

A infecção oral, assim como uma cirurgia dento-alveolar, pode diminuir o valor de pH local do osso e levar à liberação e ativação de níveis tóxicos de bifosfonatos, após o que as influências nos osteoclastos (suprimindo a remodelação óssea), nas células imunes (gerando processos infecciosos), na angiogênese (gerando isquemia) e na mucosa (promovendo toxicidade dos tecidos moles) resultam na predisposição pelo surgimento da $\mathrm{OMRB}^{4}$.

A característica pelo exclusivo acometimento dos ossos gnáticos, principalmente pela mandíbula é intrigante. Apesar de outros medicamentos também estarem associados à osteonecrose maxilomandibular, citando-se o agente biológico antirreabsortivo anti-RANK, diferentes tipos de imunomoduladores e alguns antiangiogênicos Anti-VEGF e Anti-TKIs, os Bifosfonatos são considerados os maiores causadores desta patologia, podendo- se explicar isto pelo fato de que estes fármacos são abundantes nos ossos com elevadas taxas de remodelação, como no processo alveolar. Isso parece ser mais intenso que em outros ossos, é provável que, diferentemente de outras drogas antirreabsortivas, os bisfosfonatos se acumulem fisicamente no osso alveolar de forma mais elevada4;19;18;10;11;16;20. 


\section{Conclusões}

A complexidade da fisiopatologia da osteonecrose maxilomandibular relacionada a bifosfonatos é um grave problema de saúde, exigindo que o CirurgiãoDentista compreenda suas causas, consequências e condutas terapêuticas, sendo imprescindível a tomada de medidas preventivas como o condicionamento bucal prévio ao tratamento com bifosfonatos, destacando-se os procedimentos restauradores, endodônticos, periodontais e cirúrgicos.

Surgindo a lesão, deve-se avaliar o seu estado para a melhor tomada de decisão, havendo indicação de terapia conservadora para os achados precoces em pequenas lesões, incluindo o alívio da dor e o uso de antibióticos tópicos e sistêmicos para limitar a infecção secundária e prevenir a disseminação do osso exposto, assim como realizar o desbridamento cirúrgico para o tratamento da doença avançada e não responsiva. O uso dessas medidas gera uma tendência ao sucesso do tratamento para tais casos.

\section{Referências}

1. Mercer E, Norton T, Woo S, Treister N, Dodson TB, Solomon DH. 91 Osteoporosis Patients Affected With Bisphosphonate Related Osteonecrosis of the Jaw: A Case Series. Calcif Tissue Int. 2013;93(3):241-8.

2. Pan W-L, Chen P-L, Lin C-Y, Pan Y-C, Ju Y-R, Chan C-P, et al. Strontium ranelate treatment in a postmenopausal woman with osteonecrosis of the jaw after longterm oral bisphosphonate administration: a case report. Clin Interv Aging [Internet]. 2017; Volume 12:1089-93.

3. Kang B, Cheong S, Chaichanasakul T, Bezouglaia O, Atti E, Dry SM, et al. Periapical Disease and Bisphosphonates Induce Osteonecrosis of the Jaws in Mice. J Bone Miner Res . 2013;28(7):1631-40.

Voss P, Ludwig U, Poxleitner P, Bergmaier V, El-Shafi N, Elverfeldt D Von, et al. Evaluation of BP-ONJ in osteopenic and healthy sheep: Comparing ZTE-MRI with mCT. Dentomaxillofacial Radiol. 2016;45(4): 3-7.

Conte-Neto N, Bastos AS, Spolidorio LC, Marcantonio RA, Marcantonio E. Oral bisphosphonate-related osteonecrosis of the jaws in rheumatoid arthritis patients: a critical discussion and two case reports. Head Face Med [Internet]. 
2011;7(1):7.

6. Diniz-Freitas M, López-Cedrún JL, Fernández-Sanromán J, García-García A, Fernández-Feijoo J, Diz-Dios P. Oral bisphosphonate-related osteonecrosis of the jaws: Clinical characteristics of a series of 20 cases in Spain. Med Oral Patol Oral Cir Bucal. 2012;17(5) 751-8.

7. Molon RS De, Hsu C, Bezouglaia O, Dry SM, Pirih FQ, Soundia A, et al. Rheumatoid arthritis exacerbates the severity of osteonecrosis of the jaws (ONJ) in mice. A randomized, prospective, controlled animal study. 2016;31(8):1596607.

8. Vidal-Real C, Pérez-Sayáns M, Suárez-Peñaranda JM, Gándara-Rey JM, GarcíaGarcía A. Osteonecrosis of the jaws in 194 patients who have undergone intravenous bisphosphonate therapy in Spain. Med Oral Patol Oral Cir Bucal. 2015;20(3):267-72.

9. Li Y, Xu J, Mao L, Liu Y, Gao R, Zheng Z, et al. Allogeneic Mesenchymal Stem Cell Therapy for Bisphosphonate-Related Jaw Osteonecrosis in Swine. Stem Cells Dev. 2013;22(14):2047-56.

10. Ruggiero SL, Dodson TB, Fantasia J, Goodday R, Aghaloo T, Mehrotra B, et al. American association of oral and maxillofacial surgeons position paper on medication-related osteonecrosis of the jaw - 2014 update. J Oral Maxillofac Surg [Internet]. 2014;72(10):1938-56.

11. Wan, J.T., Sheeley, D.M., Somerman, M.J. et al. Mitigating osteonecrosis of the jaw (ONJ) through preventive dental care and understanding of risk factors. Bone Res. 2020; 8, 14: 1-12.

12. Rao NJ, Wang JY, Yu RQ, Leung YY, Zheng LW. Role of Periapical Diseases in Medication-Related Osteonecrosis of the Jaws. Biomed Res Int. 2017;1-8.

13. Howie RN, Borke JL, Kurago Z, Daoudi A, Cray J, Zakhary IE, et al. A Model for Osteonecrosis of the Jaw with Zoledronate Treatment following Repeated Major Trauma. PLoS One. 2015;10(7):1-16.

14. Mori M, Koide T, Matsui Y, Matsuda T. A case of early detection of bisphosphonate-related osteonecrosis of the jaw. Indian J Pharmacol [Internet]. 2015;47(3):334.

Robert E. Marx, DDS. Miami, FL. Pamidronate (Aredia) and zoledronate (zometa) induced avascular necrosis of the jaws: a growing epidemic. J Oral Maxillofac Surg 61:1115-1118, 2003.

Eguia A, Bagán-Debón L, Cardona F. Review and update on drugs related to the development of osteonecrosis of the jaw. Medicina Oral, Patologia Oral y 
Cirugia Bucal. 2020 Jan;25(1):71-83.

17. Paper AP. American Association of Oral and Maxillofacial Surgeons Position Paper on Bisphosphonate-Related Osteonecrosis of the Jaws. J Oral Maxillofac Surg. 2007;65(3):369-76.

18. Khan AA, Morrison A, Hanley DA, Felsenberg D, McCauley LK, O'Ryan F, et al. Diagnosis and management of osteonecrosis of the jaw: A systematic review and international consensus. J Bone Miner Res. 2015;30(1):3-23.

19. Oliveira C, Brizeno L, de Sousa F, Mota M, Alves A. Osteonecrosis of the jaw induced by receptor activator of nuclear factor-kappa B ligand (Denosumab) Review. Med Oral Patol Oral y Cir Bucal. 2016;21(4):431-9.

20. AlDhalaan NA, BaQais A, Al-Omar A. Medication-related Osteonecrosis of the Jaw: A Review. Cureus. 2020;12(2):1-11. 